\title{
Comparison dietary assessment methods in Sri Lankan adults: use of 24-hour dietary recall and 7-day weighed intake
}

\author{
Ranil Jayawardena ${ }^{1,2}$
}

\begin{abstract}
s
Background: Misreporting, recalling and overestimation are common problems with dietary assessment methods currently available. The 7-day weighed food record (7DWR) method involves an individual weighing of each and every food item prior to consumption, and is considered as a reference method in many situations. The present study aims to compare the energy and nutrient intakes, estimated from 7DWR and 24-hour dietary recall (24DR), among a group of adults.

Methods: One hundred adults were randomly selected from a representative sample from Sri Lanka. The 24DRs were performed on a random day and the subjects were instructed to complete 7DWR from the next day onwards. The nutrients were analyzed using Nutrisurvey Software. In addition, qualitative data were collected from 30 participants, chosen randomly, on the feasibility of the 7DWR as a dietary assessment method.

Results: A total of 76 participants completed both 24DR and 7DWR. Mean (SD) values for energy and major macronutrients were significantly lower for 24DR as compared to 7DWR. However, there was no statistically significant difference in energy percentages for macronutrients, between the two methods. Several participants reported difficulties in using the 7DWR method and some reported an alteration in their diet pattern when this method was used.

Conclusion: Results obtained from 24DR method under-estimated the nutrient intakes as compared to the 7DWFR method.

Keywords: Dietary assessment methods, 24-hour dietary recall, 7-day weighed intake, Sri Lanka, Health adult and dietary intake
\end{abstract}

\section{Background}

Assessment of dietary intake among different populations is essential to monitor ongoing nutritional transition, and for development of appropriate interventions. However, different methods have been used to measure dietary intake at a population level, and there is no agreement on which tool best reflects the habitual dietary intake pattern [1]. Single or multiple 24-hour dietary recalls (24DR) are widely used for national level nutritional surveys [2, 3]. However, the 24 DR method has its' own limitations, such as misreporting, recall bias and poor representation of the

\section{Correspondence: ranil7@gmail.com}

${ }^{1}$ Department of Physiology, Faculty of Medicine, University of Colombo, Colombo, Sri Lanka

${ }^{2}$ Institute of Health and Biomedical Innovation, Faculty of Health, Queensland University of Technology, Brisbane, QLD, Australia usual intake [4]. Therefore, some developed countries have used the 7-day weighed record (7DWR) as a reference method to overcome many limitations of the dietary recalls [5]. However, the weighed intake method also has its own limitations, such as higher cost, substantial subject burden and impracticality for use among a larger sample [5].

Sri Lanka is a low-middle income country undergoing a nutritional transition. Prevalence of diabetes, metabolic syndrome, hypertension and obesity have reached epidemic levels [6-9]. On the other hand, nutrient deficiencies are also highly prevalent in some groups [10]. Information of energy and nutrient intake in a population is essential for development of public health policies and guidelines. Previously, the 24DR method has been used to assess the energy and nutrient intake among Sri Lankan adults in a national 
level nutrition audit [2]. Therefore, the aim of the present study is to compare a single 24-hour dietary recall with a 7-day weighed intake method among a group of Sri Lankan adults. In addition, the feasibility of the weighed record method, as a practical tool to measure the habitual intake pattern will be determined.

\section{Methods}

One hundred free-living adults (20-60 years) were randomly selected from a Sri Lanka diabetes and cardiovascular study [6]. After obtaining written informed consent, a random 24-hour dietary recall was conducted individually, by a trained nutritionist. Both weekdays and weekends were randomly selected for the interviews. Subjects were asked to recall all foods and beverages consumed over the past 24-hour time period. Standard household utensils and food portion size photographs were demonstrated to obtain information on portion sizes. Following the 24-hour DR, information on the usual intake pattern was obtained using the 7-day weighed intake method, by using kitchen scales (Tanita KD 320, Japan). A demonstration on the use of the kitchen scales was given to the participants and they were encouraged to operate the kitchen scales before the actual data collection was initiated. Basic instructions were: 1) Switch on the scale and wait for zero. 2) Before adding the food, record the weight of the empty container. 3) Add the food items and write down the weight and the details of the foods and repeat the same procedure until all food items are weighed and recorded. 4) Finally, record the weight of the leftovers. An instruction leaflet, designed in the local language was given to the subjects and a 24-hour hotline was provided to get further clarifications. Modified NutriSurvey 2007 (EBISpro, Germany) nutrient analysis software was used to analyze energy and major nutrients, using both diet assessment methods.

A qualitative approach, using in-depth interviews was undertaken on 30 participants, chosen randomly from the main sample. Subjects were asked to reflect upon their practical experiences (mainly difficulties) while following the 7DWR method. Common responses were reported. We used an interview guide for the qualitative part of the study. An example question is "what are the main practical issues you have faced during weighing your diet?"

The present study was conducted according to the guidelines laid down in the Declaration of Helsinki and was approved by the Ethical Review Committee, Faculty of Medicine, University of Colombo, Sri Lanka.

\section{Statistics}

Means (SD) of data were calculated for both 24DR and 7DWR methods. Data were observed for normal distribution. To test for significant differences between the two methods, a student $t$-test was applied. All statistical analyses were performed using the Statistical Package for the Social Sciences (SPSS) version 16.0 for Windows and a probability level of $p<0.05$ was used to indicate statistical significance. The qualitative data were analyzed using NVIVO version 10.0 (QSR International, Southport, UK).

\section{Results}

A total of 76 participants completed both 24DR and 7DWRs. Sixty-five were women and the majority were Sinhalese $(n=69), 4$ were Tamils and 3 were Moors. Mean (SD) age was 45.8 (8.1) years, and average BMI was $23.6(4.6) \mathrm{kg} / \mathrm{m}^{2}$. Thirty-eight participants were from rural areas, 30 were from urban areas and 8 were from the estate-sector (tea and rubber plantation areas).

Mean (SD) energy intake from 24DR was 1483.3 (363.3) $\mathrm{kcal} / \mathrm{d}$ and corresponding values from 7DWR were $1697.9(397.6) \mathrm{kcal} / \mathrm{d}(p<0.05)$. Similarly, a significantly lower intake of protein (48.2 g vs. $53.4 \mathrm{~g})$, fat (34.4 g vs. $39.4 \mathrm{~g})$ and carbohydrates (252.9 g vs. $301.8 \mathrm{~g})$ were reported from 24DR as compared to 7DWR. Mean consumption of dietary fiber was also significantly lower, when the 24DR method was used, as compared to the 7DWR (12.5 g vs. $14.1 \mathrm{~g})$. However, the energy percentages of macronutrients were not significantly different between the two methods (Table 1).

\section{Qualitative data on 7DWR}

The common responses of the participants are presented in Fig. 1. Twelve out of thirty found no difficulties in using the 7DWR method to measure their food intake. However, several reported (11/30) alterations in their habitual diet, when the 7DWR was used. There was a reduction in the consumption of foods consumed in between meals $(n=7)$ and outside food $(n=6)$. Dishes consumed as a part of the main meals in Sri Lanka are usually water based, in the form of curries, so few participants faced difficulties in measuring the exact weight of the curries that were consumed. Some participants faced difficulties $(n=5)$ with the use of the weighing scale and provided inaccurate data mainly due to lack of knowledge of international units (kilograms), numeracy and low technical knowledge. Measuring and recording the food items consumed, was a time consuming process and was the main reason in causing subject burden. Males were reluctant to participate in the study, due to their work commitment and inability to carry and use the scales at their respective work places. Although there were several reasons for subject burden when the 7DWR was used, measuring food before consumption was helpful to increase awareness of their own food intake.

\section{Discussion}

Single or multiple 24DRs are used in dietary surveys in many countries [2.3]. However the present study showed 
Table 1 Comparison of energy and nutrient values of 24DR and 7DWR

\begin{tabular}{|c|c|c|c|c|c|}
\hline \multirow{2}{*}{$\begin{array}{l}\text { Energy and nutrients } \\
(n=76)\end{array}$} & \multicolumn{2}{|c|}{ 24-hour recalls (24DR) } & \multicolumn{2}{|c|}{ Intake from 7-day diet diary (7DWR) } & \multirow[b]{2}{*}{$P$ value } \\
\hline & Mean & SD & Mean & SD & \\
\hline Energy (kcal/d) & 1483.3 & 363.3 & 1697.9 & 397.6 & 0.031 \\
\hline Protein (g) & 48.2 & 15.8 & 53.4 & 11.0 & 0.026 \\
\hline Fat (g) & 34.6 & 13.3 & 39.4 & 12.9 & 0.040 \\
\hline Carbohydrate (g) & 252.9 & 70.3 & 301.8 & 75.7 & 0.017 \\
\hline Dietary fiber(g) & 12.5 & 7.4 & 14.1 & 9.4 & 0.023 \\
\hline$\%$ energy from fat & 19.8 & 4.8 & 19.8 & 4.1 & 0.89 \\
\hline$\%$ energy from protein & 13.2 & 4.7 & 12.3 & 4.4 & 0.67 \\
\hline$\%$ energy from carbohydrate & 67.0 & 6.8 & 67.8 & 4.8 & 0.84 \\
\hline
\end{tabular}

that 24DRs under-reported the total energy and nutrient consumption, although there was no affect on the percentage of energy distribution, when the two methods were compared. It is always a challenge to select a suitable dietary assessment method. Under-reporting of food consumption is a well-recognized phenomenon common to dietary assessment methods [11].

All dietary assessment methods have their pros and cons [1]. To my knowledge, this is the first time that the 7DWR method was used in Sri Lanka to determine dietary intake and assess the feasibility of this method by qualitative assessment. Many studies have not found any significant burden when the 7DWR method is used, however, some studies have reported alterations in the habitual dietary intake pattern, by limiting certain food items, because of a high subject burden caused by measuring and recording individual food items. Bathalon et al. reported 24DR under reported compared to 7DWR and total energy expenditure estimated from doubly-labeled water analysis in a group of healthy postmenopausal women [12]. Therefore, it is likely that the 24DR method showed under-reporting due to various reasons such as

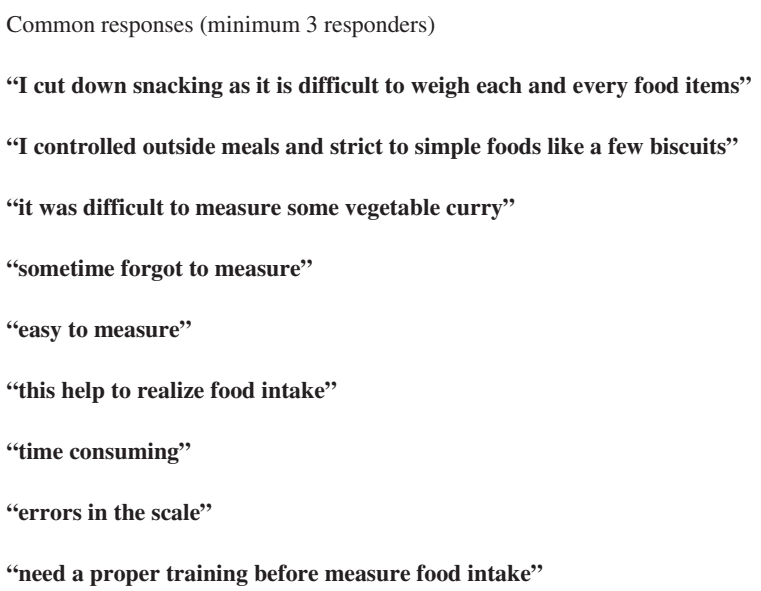

Fig. 1 Quotes obtained from interviewed participants on use of weighed food records recall bias and social desirability. Previous studies have shown that the 24DR method may under report among Sri Lankan adults. Rathnayake's study on dietary diversity among the elderly showed a mean calorie intake of 951 (300) $\mathrm{kcal} / \mathrm{d}$ [13]. This is considerably low for an average elderly individual. Mean energy intakes of Malaysian men and women were 1776 and $1447 \mathrm{kcal} / \mathrm{d}$ respectively. Both were less than $75 \%$ of the Reference Nutrient Intake (RNI) [3].

Qualitative data showed practical problems associated with measuring food intake using 7DWR. Since there is a considerable subject burden on weighing food items, 7DWR may not be suitable for large studies and especially for male populations. Therefore, future researchers should provide other options such as food photography methods e.g. "Remote Food Photography Method (RFPM)" to reduce their subject's burden. Measurement of food intake before eating, also gives a fair idea on the weight and portion size of food items, and this can influence the consumption patterns. To avoid technical difficulties, a considerable amount of training is important prior to use of scales for obtaining reliable information.

\section{Limitations}

Firstly, this study may not represent dietary habits and subject perception on 7DWR among Sri Lankan adults due to the small sample size and low response rate. Male participation was very low and most of the participants were housewives. However, our primary aim was to compare energy and nutrient intake using two dietary assessment methods and observe the difficulties in applying the 7DWR method compared to the 24DR. Secondly, a complete database on the nutritional composition of Sri Lankan dishes is not available. Although we used accurate food composition values as far as possible, macronutrient values for some Sri Lankan dishes were not $100 \%$ accurate [14]. Thirdly, none of these methods is considered as a reference method to measure energy intake. In an ideal situation, the doubly labeled water technique, which is considered the gold standard for measurement of 
energy expenditure should be used and the intake data obtained from a dietary method should be compared for inaccuracies.

\section{Conclusion}

In summary, this study showed that the 24DR method under-estimated nutrient intakes compared to the 7DWR method. However, the 7DWR method has its own limitations; thus, it is important to assess the feasibility of a dietary assessment method before it is used for a particular study population. Qualitative data provided valuable information on the feasibility of the 7DWR method.

\section{Abbreviations}

24DR: 24 - hour dietary recall; 7DWR: 7 - day weighed food records;

SD: standard deviation.

\section{Competing interests}

The author declares that he has no competing interests.

\section{Author's contribution}

RJ contributed to the designing of the study, data collection, data analysis and drafted the manuscript.

Received: 22 June 2015 Accepted: 19 March 2016

Published online: 21 March 2016

\section{References}

1. Bingham SA et al. Comparison of dietary assessment methods in nutritional epidemiology: weighed records v. $24 \mathrm{~h}$ recalls, food-frequency questionnaires and estimated-diet records. Br J Nutr. 1994;72(4):619-43.

2. Jayawardena $\mathrm{R}$ et al. Energy and nutrient intakes among Sri Lankan adults. Int Arch Med. 2014;7(1):34

3. Mirnalini Jr K et al. Energy and Nutrient Intakes: Findings from the Malaysian Adult Nutrition Survey (MANS). Malays J Nutr. 2008;14(1):1-24.

4. Raina SK. Limitations of 24-hour Recall Method: Micronutrient Intake and the Presence of the Metabolic Syndrome. N Am J Med Sci. 2013;5(8):498.

5. Swan G. Findings from the latest National Diet and Nutrition Survey. Proc Nutr Soc. 2004;63(04):505-12.

6. Katulanda P et al. Prevalence and projections of diabetes and pre-diabetes in adults in Sri Lanka-Sri Lanka Diabetes, Cardiovascular Study (SLDCS). Diabet Med. 2008;25(9):1062-9

7. Katulanda P et al. The prevalence, predictors and associations of hypertension in Sri Lanka: a crosssectional population based national survey. Clin Exp Hypertens. 2014;36(7):484-91.

8. Katulanda $\mathrm{P}$ et al. Metabolic syndrome among Sri Lankan adults: prevalence, patterns and correlates. Diabetol Metab Syndr. 2012;4(1):24.

9. Katulanda $\mathrm{P}$ et al. Prevalence of overweight and obesity in Sri Lankan adults. Obes Rev. 2010;11(11):751-6.

10. Jayatissa R, Ranbanda RM. Prevalence of challenging nutritional problems among adolescents in Sri Lanka. Food Nutr Bull. 2006;27(2):153-60.

11. Holmes B, Dick K, Nelson M. A comparison of four dietary assessment methods in materially deprived households in England. Public Health Nutr. 2008;11(05):444-56.

12. Bathalon GP et al. Psychological measures of eating behavior and the accuracy of 3 common dietary assessment methods in healthy postmenopausal women. Am J Clin Nutr. 2000;71(3):739-45.

13. Rathnayake K, Madushani PAE, Silva K. Use of dietary diversity score as a proxy indicator of nutrient adequacy of rural elderly people in Sri Lanka. BMC Res Notes. 2012;5(1):1-6.

14. Jayawardena $\mathrm{R}$ et al. Development of a food frequency questionnaire for Sri Lankan adults. Nutr J. 2012;11:63-3
Submit your next manuscript to BioMed Central and we will help you at every step:

- We accept pre-submission inquiries

- Our selector tool helps you to find the most relevant journal

- We provide round the clock customer support

- Convenient online submission

- Thorough peer review

- Inclusion in PubMed and all major indexing services

- Maximum visibility for your research

Submit your manuscript at www.biomedcentral.com/submit 